\title{
Measurement of Oral Health Literacy Level Among Bangladeshi Adults Seeking Dental Care and Its Relationship With Socio-demographic Characteristics
}

\author{
*SZ Mahmud ${ }^{1}$, MS Amin ${ }^{2}$, MA Tarafder ${ }^{3}$, SM Hossain ${ }^{4}$ \\ 1*Shaikh Zakir Mahmud, Senior Medical Officer (Dental Surgeon), Ibrahim General Hospital Mirpur, Dhaka, Bangladesh \\ ${ }_{2}^{2}$ Mohammad Shoheilul Amin, Assistant Professor, Pioneer Dental College, Dhaka, Bangladesh \\ ${ }^{3}$ Monowar Ahmad Tarafder, Professor, Department of Community Medicine, Zainul Haque Sikder Women's \\ Medical College, Dhaka, Bangladesh \\ ${ }^{4}$ Sarder Mahmud Hossain, Professor \& Head, Department of Public Health, Northern University Bangladesh \\ *Corresponding Author
}

Date of submission: 03 Sept 2016

Date of acceptance: 17 Dec 2015

\begin{abstract}
Background: The important role of socio-economical factors in oral health and disease has been identified. Oral health literacy is associated with inaccurate knowledge about preventive measures such as proper brushing, dental care visits and oral health-related quality of life. Like general health literacy, oral health literacy incorporates the capacity a person has to learn and use information about oral health in making decisions about their oral health. Objective: This analytic type of cross sectional study was conducted to measure the Oral Health literacy Level (OHLL) and its relationship with socio-demographic characteristics among Bangladeshi adults seeking dental care.

Materials and Methods: A total 100 subjects attended at the out patient's Dental Department of Ibrahim General Hospital-Mirpur for routine dental checkup over a period of six months from August 2014 to January 2015 fulfilled the eligibility criteria were selected consecutively. Pre-tested semi structured interviewer administrated questionnaires were used to collect the information. Cross tabulations and associations were determined by using the Chi-square test where applicable.

Results: The mean oral health literacy score was 15.51 out of 30 and SD \pm 4.806 with $7 \%$ of respondents had very poor OHLL, $49 \%$ had poor OHLL, $41 \%$ had fair and 3\% had excellent oral health literacy level. Oral health literacy level found more in age group of 30-39 years. The majority of excellent oral health literacy level was from graduate and above group. In addition, patients' sex, marital status and occupation were significantly associated with oral health literacy level as the P-values were $0.008,0.002$ and 0.021 respectively.

Conclusion: Patients with inadequate oral health literacy were consistently more likely to report poor oral health than patients with adequate literacy skills. Future studies are needed to determine whether this framework is supported by empirical data and leads to improvements in oral health and reductions in health disparities.
\end{abstract}

Key Words: Dental care, diabetes mellitus, socio-demographic characteristics, oral health literacy

\section{Introduction}

In the oral health context, literacy can be considered as the skills necessary for people to understand the causes of poor oral health, to learn and adopt fundamental aspects of positive oral self-care behaviors, to communicate with oral health care providers, to place their names on dental treatment waiting lists or organize appointments, to find their way to the dental clinic, to fill out the necessary forms and to comply with any required regimes, including follow-up appointments and compliance with prescribed medication. Lack of oral health literacy (OHL) can create a significant barrier to AKMMC J 2016; 7(1) : 34-39 prevent oral disease and to promote oral health. ${ }^{1}$ The general perception that dentistry is expensive keeps many people away from the registered professionals on one hand, while on the other hand making them hostage to the services of nonregistered lay practitioners. The result is that the people of Bangladesh, in general, have a very low level of awareness regarding oral health and hygiene. ${ }^{2}$ Although much is known about health literacy in the medical context, little is known about oral health literacy (OHL) and its relationship to clinical conditions, patients' 
Measurement of Oral Health Literacy Level Among Bangladeshi Adults subjective assessments, and OHL's perceived impacts on daily life in the community. A working group defined OHL as "the degree to which individuals have the capacity to obtain, process, and understand basic oral health information and services needed to make appropriate health decisions." 3

Oral health literacy is the new imperative for better oral health as health literacy is now considered a determinant of health. ${ }^{4}$ But the problem is when one cannot make appropriate health decisions and the burden of oral disease is particularly high for the disadvantaged and poor population groups in both developing and developed countries. In addition to poor living conditions, the major risk factors relate to unhealthy lifestyles (i.e. poor diet, nutrition and oral hygiene and use of tobacco and alcohol), and limited availability and accessibility of oral health services. ${ }^{5}$ Health literacy encompasses several abilities including word recognition, reading comprehension, communication skills, and conceptual knowledge. To date, conceptual knowledge has not been included in oral health literacy research. ${ }^{6}$ Researchers hypothesize that an individual's health literacy is represented by a constellation of skills and abilities, including word recognition, reading comprehension, communication proficiency, and conceptual knowledge. ${ }^{7}$ Lack of education along with traditional beliefs and socio-cultural factors lead to development of false perceptions and myths. The concept of dental myth usually emerges from false traditional beliefs and non-scientific knowledge. This is embedded in the psyche of future generations over a period of time, and, thus, creates hindrance in the recognition of scientific and contemporary dental treatment. ${ }^{8}$

The current study aimed at measuring the proportion of oral health literacy level and perceived behavior regarding oral healthcare and its association with socio-demographic characteristics.

\section{Materials \& Methods}

This analytic type of cross sectional study was conducted to assess the Oral Health literacy Level (OHLL) and its relationship with socio-demographic characteristics among Bangladeshi adults attended at out patient's Dental Department of Ibrahim General Hospital-Mirpur. The study was carried out from August 2014 to January 2015 among 100 subjects.
To get the target sample quickly non randomized purposive sampling technique was followed by using a pre tested semi-structured questionnaires. Only adult man and women of age 18-60 years were included in this study. On the other hand, those who refused to provide informed consent and who were below 18 or above 60 years were excluded from the study.

Oral health literacy was measured using an oral health adult literacy questionnaire (OHL-AQ). The questionnaire consisted of 5 subgroups (General health, General Oral Health, Factors that can affect oral health, Oral hygiene maintenance, Oral health seeking behaviour) to find out the outcome variable with total 30. The relevant aspect of data coded into point ' 1 ' for no dental problem or good practice and ' 0 ' for presence of any oral condition or negative attitude and behavior. Possible maximum score was 30 points. Score 24-30 set as excellent oral health literacy level, 17-23 fair, 10-16 poor and below 10 score set as very poor oral health literacy level. Level of oral health literacy was the main focus of the study. Data were checked, cleaned and edited properly before analysis. The data were sorted and analyzed by using the software SPSS version 11.5. The analyzed data were presented in the form of frequency distribution tables. Cross tabulations and associations were determined by using the Pearson Chi-square test where applicable.

\section{Results}

The respondents' mean oral health literacy score was 15.51 and SD \pm 4.806 . Most of respondents were found to more oral health literacy in 30-39 years age group (Figure 1).

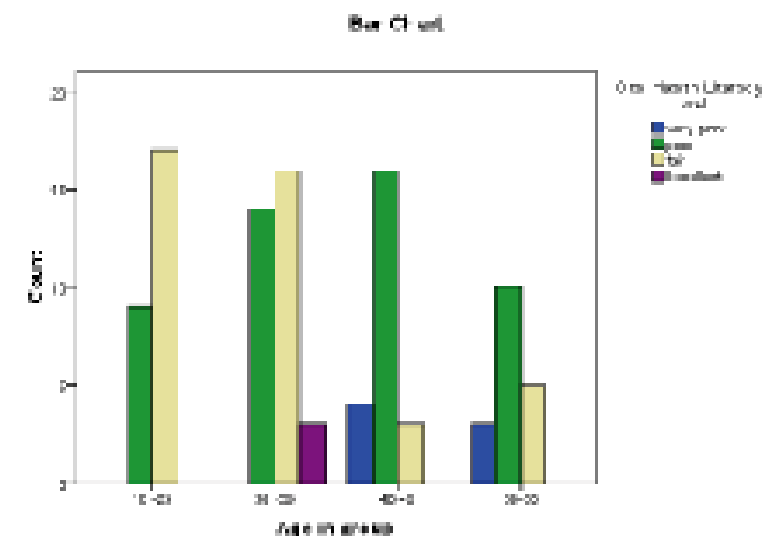

Figure 1: Bar Chart showing distribution of the patients according to age group in relation to oral health literacy level $(n=100)$ 
The distribution of the patients according to the proportions of oral health literacy level is shown in the Table I. Among them, 7\% had very poor OHLL, $51 \%$ had poor OHLL, $39 \%$ had fair and $3 \%$ had excellent oral health literacy level. Moreover, out of 32 male, $17 \%$ had fair, $13 \%$ had poor and $2 \%$ had excellent OHLL. What's more, out of 68 female, $38 \%$ had poor OHLL followed by $22 \%$ fair, $7 \%$ very poor and only $1 \%$ had excellent OHLL. In addition, a significant association found between patients' sex and oral health literacy level $(\mathrm{P}=0.008)$.

Table-I: Distribution of the patients according to gender in relation to the proportions of oral health literacy level $(n=100)$

\begin{tabular}{|c|c|c|c|c|}
\hline \multirow[b]{2}{*}{ Oral health literacy level (OHLL) } & \multicolumn{2}{|c|}{ Sex } & \multirow[b]{2}{*}{ Total } & \multirow[b]{2}{*}{ p-valuea } \\
\hline & Male & Female & & \\
\hline Excellent & 2 & 1 & 3 & \\
\hline Fair & 17 & 22 & 39 & \\
\hline Poor & 13 & 38 & 51 & $0.008^{*}$ \\
\hline Very poor & 0 & 7 & 7 & \\
\hline Total & 32 & 68 & 100 & \\
\hline \multicolumn{5}{|l|}{ a Pearson Chi-Square Test } \\
\hline * Statistically significant association & $0.05)$ & & & \\
\hline
\end{tabular}

The distribution of the patient according to diabetes mellitus in relation to oral health literacy level is shown in the Table II. Among the respondents, 74\% had diabetes mellitus, and the rest $26 \%$ were absence of diabetes mellitus.

Table-II: Distribution of patients according to the history of Diabetes Mellitus $(n=100)$

\begin{tabular}{ccc}
\hline Diabetes Mellitus & Frequency & Percent (\%) \\
\hline Yes & 74 & 74.0 \\
No & 26 & 26.0 \\
Total & 100 & 100.0
\end{tabular}

Results were expressed as frequency (\%)

The distribution of the patients according to oral health related behavior, in relation to in relation to oral health literacy level is shown in Table III. Here only $32 \%$ patients cleaned their teeth twice a day and
$27 \%$ knew how to brush their teeth. Moreover, $58 \%$ of the patients visited to the dentist whereas $11 \%$ visited to dental technician or quack for their dental problem. Furthermore, 33\% participants believed about myth in dentistry. Most of patients' common myths were "Extraction of upper teeth will affect vision" and "Charcoal, salt; rice husk and tobacco powder cleans better than toothpaste".

Table-III: Distribution of the patients according to oral health care related behavior, in relation to oral health literacy level $(n=100)$

\begin{tabular}{lcc}
\hline Characteristics & Frequency & Percent (\%) \\
\hline $\begin{array}{l}\text { Clean teeth twice a day } \\
\text { No }\end{array}$ & 68 & 68.0 \\
Yes & 32 & 32.0 \\
$\begin{array}{l}\text { Know the technique of tooth } \\
\text { brushing }\end{array}$ & & \\
No & 73 & 73.0 \\
Yes & 27 & 27.0 \\
Visiting dentist for dental & & \\
problem & & \\
No & 42 & 42.0 \\
Yes & 58 & 58.0 \\
Visiting dental technician or & & \\
quack for dental problem & & \\
No & 89 & 89.0 \\
Yes & 11 & 11.0 \\
$\begin{array}{l}\text { Believed about the myth in } \\
\text { dentistry }\end{array}$ & & \\
No & 67 & 67.0 \\
Yes & 33 & 33.0 \\
\hline
\end{tabular}

The frequency of patients' socio-demographic characteristics in relation to oral health literacy level is provided in Table IV. Age range was 18 to 60 years. Middle aged people suffered more from dental problems. Mean age of the patients were $37.01 \pm 10.457$ years. Among them females were common (68\%). Muslim participants were high in number $(95 \%)$. Most of the patients were married (72\%). Education level was widespread with $11 \%$ had no formal education, $19 \%$ went to primary school, $17 \%$ had SSC level education, $26 \%$ HSC and $20 \%$ had more than HSC level education. $46 \%$ were housewives. $43 \%$ earns less than 5000 taka per month while $16 \%$ earns more than 15000 taka per month. Mean monthly income of the patients were $5338.89 \pm 1358.813$ taka. Maximum (85\%) were from rural area. Moreover, patients' sex, marital status and occupation were 
significantly associated with oral health literacy level as the P-values were $0.008,0.002$ and 0.021 respectively. In addition, highly significant associations were found between patients' age, education, monthly income and oral health literacy level $(\mathrm{P}=0.000)$.

Table-IV: Distribution of the patients according to socio-demographic characteristics, in relation to oral health literacy level $(n=100)$

\begin{tabular}{|c|c|c|c|}
\hline Characteristics & Frequency & Percent (\% & $\overline{P-\text { value }^{\mathrm{a}}}$ \\
\hline Age & Mean $=37.01 \pm 10.457$ & & $0.000^{*}$ \\
\hline 18- 29 Years & 26 & 26.0 & \\
\hline 30 - 39 Years & 33 & 33.0 & \\
\hline 4049 Years & 23 & 23.0 & \\
\hline 5060 Years & 18 & 18.0 & \\
\hline Sex & & & $0.008^{*}$ \\
\hline Male & 32 & 32.0 & \\
\hline Female & 68 & 68.0 & \\
\hline Religion & & & 0.526 \\
\hline Muslim & 95 & 95.0 & \\
\hline Hindu & 5 & 5.0 & \\
\hline Marital status & & & $0.002 *$ \\
\hline Single & 18 & 18.0 & \\
\hline Married & 72 & 72.0 & \\
\hline Widow & 10 & 10.0 & \\
\hline Education & & & $0.000 *$ \\
\hline Illiterate & 11 & 11.0 & \\
\hline Primary & 19 & 19.0 & \\
\hline Secondary & 7 & 7.0 & \\
\hline SSC & 17 & 17.0 & \\
\hline HSC & 26 & 26.0 & \\
\hline Under Graduate or above & 20 & 20.0 & \\
\hline Occupation & & & $0.021^{*}$ \\
\hline Unemployed & 12 & 12.0 & \\
\hline Service & 23 & 23.0 & \\
\hline Farmer & 4 & 4.0 & \\
\hline Day labour & 2 & 2.0 & \\
\hline Business & 12 & 12.0 & \\
\hline House wife & 46 & 46.0 & \\
\hline Others & 1 & 1.0 & \\
\hline \multicolumn{3}{|c|}{ Monthly Income (Taka)Mean $=\mathbf{5 3 3 8 . 8 9} \pm 1358.813$} & $0.000^{*}$ \\
\hline$<5000$ & 43 & 43.0 & \\
\hline 5001- 10000 & 29 & 29.0 & \\
\hline $10001-15000$ & 12 & 12.0 & \\
\hline $15000>$ & 16 & 16.0 & \\
\hline Living area & & & 0.432 \\
\hline Rural & 85 & 85.0 & \\
\hline Urban & 15 & 15.0 & \\
\hline
\end{tabular}

aPearson Chi-square test

*Statistically significant association $(P<0.05)$

\section{Discussion}

According to the 2003 National Adult Assessment of Literacy, about $22 \%$ adults had only basic literacy skills, and as many as $14 \%$ of adults had below-basic abilities. ${ }^{9}$ A short version of the Rapid Estimate of Adult Literacy in Dentistry-30 (REALD-30) was done where about 29\% of the sample scored below 22 on the 30-point test, a score that defined as a low literacy level. Those with incorrect knowledge and fair-to-poor oral health status were more likely to have a low literacy level than were their reference groups. ${ }^{10}$ The data in the present study showed that $7 \%$ had very poor OHLL, $51 \%$ had poor OHLL, 39\% had fair and $3 \%$ had excellent oral health literacy level.

Diabetes mellitus affects all age groups, but is more common in adults. The World Health Organization (WHO) has recently declared it to be a pandemic. Various inflammatory diseases and soft tissue pathologies in oral cavities are associated with diabetes mellitus. ${ }^{11}$ Some studies report limited health literacy is associated with poor glycemic control whereas others report limited health literacy is associated with poor disease knowledge and less adherence to diabetes self-care, but is not associated with glycemic control. ${ }^{12}$ Present study showed that $74 \%$ had diabetes mellitus, and the rest $26 \%$ were absence of diabetes mellitus.

A study was done to evaluate tooth-brushing technique and oral hygiene knowledge in Rawalpindi population. The result showed that $87 \%$ brushed their teeth twice a day, only $4 \%$ knew proper technique and $43 \%$ were regular in their dental check up and follow up visits to their dentist. The overall awareness about oral hygiene knowledge was not satisfactory. ${ }^{13}$ Present study showed that 32\% cleaned their teeth twice a day, only $27 \%$ knew the technique of tooth brushing and 58\% visited to their dentist for dental check up while $11 \%$ went to dental technician or quack for their dental problem.

Communities and countries with inappropriate exposure to oral health care delivery systems are at higher risk of oral diseases, when socio-cultural determinants such as poor living conditions; low education; lack of traditions, beliefs, culture \& myths related to oral health are more prevalent. ${ }^{14}$ In 2010, a descriptive cross-sectional study was conducted where $33 \%$ of the respondents believed 
that extraction of tooth affects vision, whereas $62 \%$ believed that it is not true. ${ }^{15}$ In another study, $24.47 \%$ believed that charcoal, salt, rice husk, tobacco, etc, in powder form is better than toothpaste in cleaning teeth. ${ }^{16}$ The data of the present study showed that $67 \%$ participants believed about myth in dentistry and $33 \%$ did not believe about myths. Most of patients' believed common myths were "Extraction of upper teeth will affect vision" and "Charcoal, salt; rice husk and tobacco powder cleans better than toothpaste".

To measure oral health knowledge, a study was done among 100 low-income Baltimore adults that were categorized into three levels of knowledge (poor, fair, good). Nearly one-third of adults exhibited the lowest level. Comprehensive measures of oral health knowledge scores were significantly associated with age, education level, and annual household income. ${ }^{6}$ In this study, highly significant associations were found between patients' age, education, monthly income and oral health literacy level $(\mathrm{P}=0.000)$. In another analytic study, their mean age was 26.6 years $(\mathrm{SD} \square=\square 6.9)$. Two thirds of participants had a high school education or less, and less than one third rated their oral health as very good or excellent. ${ }^{17}$ According to the data of the present study, the mean age of the patients were 37.01 years $(\mathrm{SD}=10.45)$. About $17 \%$ had higher secondary school (HSC) education and only 3\% rated their oral health literacy as excellent.

The average oral health literacy level of Iranian adults was low. Women $(\mathrm{p}<0.001)$, younger $(\mathrm{p}<0.001)$, and better educated participants $(\mathrm{p}<0.001)$ had higher OHL scores. Disseminating evidence-based oral health care information from multiple sources including TV/radio, dentists, and other health professionals in different settings should improve public oral health literacy. ${ }^{18}$ In this study, patients' sex, marital status and occupation were significantly associated with oral health literacy level as the Pvalues were $0.008,0.002$ and 0.021 respectively.

\section{Limitations}

This study would have needed a much larger sample size in order to generalize the results in Bangladeshi population. As there was limited time frame and resources to conduct the study, we used the most convenient formula for calculate the sample size. Therefore, the sample size became smaller which may decrease the statistical power.
Other limitation of this study was the veracity of the data collection by purposive sampling method using a semi-structured questionnaire. In this investigation the homogeneity of the sample could not be ensured. For instance, respondents from the sex groups were not equal in number. Again, socioeconomic backgrounds of the patients were varied, as the family income depends upon the number of persons employed.

Oral health related behavior mentioned in the questionnaire may differ from actual behavior, as the respondents have full authority to answer the questions as they liked and there was no system for cross check. The limitation of time, resources and facilities caused problems for an in-depth study particularly for the individual researcher.

\section{Conclusion}

The current study showed that patients with inadequate oral health literacy were consistently more likely to report poor oral health than patients with adequate literacy skills. Association between oral health literacy and self-reported poor health should be interpreted cautiously. In addition, individual questions and oral health status scales need to be validated in low-literate patients to ensure that any differences in reported health do not merely result from differences in response styles between patients with low literacy and those with adequate education and knowledge. Thus, an extensive oral and dental health education is needed to raise knowledge and awareness so as to internalize the preventive oral and dental health practices into daily life style of the population. Future studies are needed to determine whether this framework is supported by empirical data and leads to improvements in oral health and reductions in health disparities.

Conflict of interest : We have no conflict of interest. 


\section{References}

1. Haridas R, Supreetha S, Ajagannanavar SL, et al. Oral health literacy and oral health status among adults attending dental college hospital in India. Journal of International Oral Health 2014; 6(6):61-66.

2. Khan AM, Ahmed SM. "Why do I have to clean teeth regularly?": Perceptions and state of oral and dental health in a low-income rural community in Bangladesh. BRAC research and evaluation 2011. RED working paper No. 20. [Internet] Available from: http://www.bracresearch.org/workingpapers/red_wp20_new.pdf [cited 2014 June 09]

3. The invisible barrier: literacy and its relationship with oral health. A report of a workgroup sponsored by the National Institute of Dental and Craniofacial Research, National Institute of Health, U.S. Public Health Service, Department of Health and Human Services. J Public Health Dent 2005, 65:174-182.

4. Horowitz AM, Kleinman DV. Oral health literacy: the new imperative to better oral health. Dent Clin North Am 2008; 52(2): 333-344.

5. Petersen PE, Bourgeois D, Ogawa H, Day SE, Ndiaye C. The global burden of oral diseases and risks to oral health. Bull World Health Organ 2005; 83(9):661-669.

6. Macek MD, Haynes D, Wells W, et al. Measuring conceptual health knowledge in the context of oral health literacy: preliminary results. J Public Health Dent 2010;70(3):197-204.

7. Nielsen-Bohlman L, Panzer A, Kindig DA, editors. Institute of Medicine of the National Academies. What is health literacy?; Committee on Health Literacy. Health literacy: a prescription to end confusion.Washington, DC: National Academy of Sciences; 2004.p. 31-58.

8. Khan SA, Dawani N, Bilal S. Perceptions and myths regarding oral health care amongst strata of low socio economic community in Karachi, Pakistan. J Pak Med Assoc 2012; 62(11): 1198-1203.

9. Kutner M, Greenberg E, Jin Y, Paulsen C. The health literacy of America's adults: Results from the 2003 national assessment of adult literacy (NCES 2006-483). U.S. Department of Education. Washington, DC: National center for education statistics; 2006.p.9-14.
10. Jones M, Lee JY, Rozier RG. Oral health literacy among adult patients seeking dental care. The Journal of the American Dental Association 2007; 138(9): 11991208.

11. AY, Al-Maskari MY, Al-Sudairy S. Oral manifestations and complications of diabetes mellitus. Sultan Qaboos Univ Med J 2011; 11(2): 179-186.

12. CY Osborn, SS Bains, LE Egede. Health literacy, diabetes self-care, and glycemic control in adults with type 2 diabetes. Diabetes Technol Ther 2010; 12(11): 913-919.

13. Bangash RY, Khan AU, Tariq KM, Rasheed D. Evaluation of tooth brushing technique and oral hygiene knowledge at AFID, Rawalpindi. Pakistan Oral \& Dental Journal 2012; 32(1): 124-127.

14. Strategies and approaches in oral disease prevention and health promotion, WHO. [Internet] Available from : http://www.who.int/oral_health/strategies/cont/en/ [cited 2015 January 19]

15. Nasir Z, Ahmed W, Iqbal F, et al. Prevalence of social myths and taboos related to dental health among general population of Rawalpindi-Pakistan. Pakistan Oral \& Dental Journal 2014; 34(3): 520-523.

16. Kumar S, Mythri H, Kashinath KR. A clinical perspective of myths about oral health: A hospital based survey. Universal Journal of Pharmacy 2014, 03(1): 3537.

17. Lee JY, Divaris K, Baker AD, Rozier RG, Vann-Jr WF. The relationship of oral health literacy and selfefficacy with oral health status and dental neglect. Am J Public Health. 2012 May; 102(5): 923-929.

18. Sistani MM, Yazdani R, Virtanen J, Pakdaman A, Murtomaa H. Oral health literacy and information sources among adults in Tehran, Iran. Community Dent Health 2013; 30(3): 178-182. 